THE effects of chronic mild prenatal stress on leukocyte infiltration into the airways was investigated in rat offspring. The chronic prenatal stress consisted of transitory and variable changes in the rat's living conditions. Offspring at adult age were actively sensitized (day 0 ) and intratracheally challenged (day 14) with ovalbumin. Bronchoalveolar lavage was performed in the offspring at $48 \mathrm{~h}$ after intratracheal challenge with ovalbumin. A significant increase in total leukocyte infiltration was observed in the nonstressed offspring group and this was associated with a marked recruitment of eosinophils without a significant effect on the influx of neutrophils and mononuclear cells. In the prenatal stressed offspring, the counts of both total leukocyte and eosinophils, as well as mononuclear cells, was increased by $50 \%$ compared to the non-stressed offspring. We provide here the first experimental evidence that chronic mild unpredictable prenatal stress produces a marked increase in the allergen-induced airway inflammation in the rat offspring.

Key words: Prenatal stress; Eosinophils; Asthma; Development; Lungs

\section{Chronic mild prenatal stress exacerbates the allergen-induced airway inflammation in rats}

\author{
Paulo J. Nogueira, ${ }^{1}$ Heloisa H. A. Ferreira, ${ }^{2}$ \\ Edson Antunes ${ }^{3}$ and Nancy A. Teixeira ${ }^{3, C A}$
}

${ }^{1}$ Departamento de Farmacologia, Instituto de Biociências, Universidade Estadual Paulista, Botucatu, SP, Brasil; ${ }^{2}$ Universidade São Francisco, Bragança Paulista, SP, Brasil; ${ }^{3}$ Departamento de Farmacologia, Faculdade de Ciências Médicas, UNICAMP, P.O. Boc 6111, 13081-970 Campinas (SP), Brasil

${ }^{\mathrm{CA}}$ Corresponding Author

Fax: (+55) 192892968

Email: airoldi@obelix.unicamp.br

\section{Introduction}

Asthma is a disease characterized by reversible episodes of bronchoconstriction and increase in airway hyper-responsiveness to various bronchoconstrictor stimuli. ${ }^{1}$ This is associated with extensive airway inflammation caused mainly by leukocyte infiltration, especially eosinophils. ${ }^{2}$ Different extrinsic stimuli such as allergens, chemical irritants, cold air, exercise and viral (or bacterial) infections trigger the airway inflammation. However, the provoking agents in intrinsic asthma are still poorly understood but certainly include abnormal responses to stress. ${ }^{3}$ Although clinical studies suggest a clear correlation between stressful events lived by the mother during pregnancy and progression of respiratory disease in the descendants, ${ }^{3,4}$ no animal model of stress so far employed has demonstrated an increased leukocyte (particularly eosinophils) infiltration in the airways as that observed in asthmatic patients. In this study we exposed pregnant rats to chronic mild unpredictable prenatal stress, ${ }^{5}$ and studied the allergeninduced airway inflammation in the female offspring. We examined therefore the total and differential (neutrophils, eosinophils and mononuclear cells) leukocyte counts in the bronchoalveolar lavage fluid from the actively sensitized female offspring at adult age.

\section{Material and methods}

\section{Animals and stress schedule}

Female nulliparous Wistar rats (200-230 g), provided by the Biology Institute Laboratory Animal Center (CEMIB) of the State University of Campinas (UNICAMP), were used. They were individually housed at $26 \pm 2{ }^{\circ} \mathrm{C}$ with food and water ad libitum on a 12-h light:dark cycle with the lights turned on at $06: 00 \mathrm{~h}$ during at least 3 days acclimatization period, at the end of which an adult male rat was placed in each cage. The date of conception (day 0 of pregnancy) was determined where sperm was detected in expelled vaginal plugs. The females were then isolated and randomly assigned to either non-stressed (control) or stressed animals. Control rats were left undisturbed throughout pregnancy except for routine animal care. The stressed group underwent a schedule of chronic mild unpredictable stress, as previously described. ${ }^{5}$ This procedure was performed, beginning on day 7 of gestation and continued through day 19. ${ }^{6}$ Briefly, the stress regime (per week) consisted of $60 \mathrm{~h}$ of food and $78.5 \mathrm{~h}$ of water deprivation, $35 \mathrm{~h}$ of continuous lighting, $24.5 \mathrm{~h}$ of cage tilting, $17 \mathrm{~h}$ of paired housing, $17 \mathrm{~h}$ in a soiled cage, $2 \mathrm{~h}$ of reduced temperature, $10 \mathrm{~h}$ of exposure to intermittent white noise $(85 \mathrm{~dB}), 1 \mathrm{~h}$ of stroboscopic lighting, $0.5 \mathrm{~h}$ of exposure to empty water bottles 
following a period of water deprivation, $2 \mathrm{~h}$ of restricted access to food, $17 \mathrm{~h}$ of exposure to a new odor, and $17 \mathrm{~h}$ of exposure to objects in the home cage. Females near parturition were visually inspected twice daily (at 09.00 and $16.00 \mathrm{~h}$ ). At birth, litters were reduced to eight pups per dam, four females and four males, when possible. The offspring were kept together with their dams and left undisturbed, except for routine animal care. On weaning, at the age of 21 days, the females were housed in a group of 10 according to the prenatal treatment until testing at adult age (75 days). Tests were performed using one to two rats per litter per test.

\section{Sensitization procedure and antigen challenge in the offspring rats}

Active sensitization against ovalbumin (OVA; ovalbumin chicken egg, Grade III, Sigma, USA) was performed by s.c. injection of $0.15 \mathrm{ml}$ solution containing $200 \mu \mathrm{g}$ of OVA and $8 \mathrm{mg} \mathrm{Al}(\mathrm{OH})_{3}$ prepared in saline. Non-sensitized offspring received only $8 \mathrm{mg}$ $\mathrm{Al}(\mathrm{OH})_{3}$. On day 14, both sensitized and nonsensitized offspring were anaesthetized with chloral hydrate $(300 \mathrm{mg} / \mathrm{kg}$, i.p.) and the trachea was exposed through a midline ventral incision of approximately $0.5 \mathrm{~cm}$ length in the neck. With the aid of a 26.5 gauge needle, $0.4 \mathrm{ml}$ of $0.25 \%$ solution of OVA was injected into the airways. Immediately after this procedure, the animals were sutured and allowed to recover from the anaesthesia. Bronchoalveolar lavage was performed $48 \mathrm{~h}$ after OVA challenge. Briefly, the animals were again anaesthetized with chloral hydrate $(300 \mathrm{mg} / \mathrm{kg}$, i.p.) and exsanguinated by cutting the abdominal aorta. The trachea was exposed and cannulated with a polyethylene tube ( $1 \mathrm{~mm}$ diameter) connected to a syringe. The lungs were washed by flushing with phosphatebuffered saline (PBS) solution containing heparin (20 IU/ml) and $0.03 \%$ serum albumin. The PBS buffer was instilled through the tracheal cannula as one $10-\mathrm{ml}$ aliquot followed by three $5-\mathrm{ml}$ aliquots. The fluid recovered after each aliquot instillation was combined and centrifuged $(1000 \mathrm{~g}$ for $10 \mathrm{~min}$ at $20^{\circ} \mathrm{C}$ ). The cell supernatant was discarded and the cell pellet was resuspended in $2 \mathrm{ml}$ of PBS buffer. The total cell numbers were counted in Türk's solution while differential counts were carried out on air-dried smears stained with May-GrünwaldGiemsa. A minimum of 400 cells was counted and classified as neutrophils, eosinophils and mononuclear cells based on normal morphological criteria. The sensitization procedure here employed is efficient, since challenge of the trachea strips with OVA $(10 \mu \mathrm{g})$ causes a significant contraction of the tissues obtained from OVA-sensitized rats, whereas strips obtained from non-sensitized animals do not show any contractile effect. ${ }^{7}$

\section{Experimental protocols}

The female offspring were initially divided into two groups: non-stressed offspring group (litters from non-stressed dams) and prenatal-stressed offspring group (litters from prenatal-stressed dams). At adult age (75 days), each of these groups was further divided into another two groups: non-sensitized (rats that received s.c. only $\left.8 \mathrm{mg} \mathrm{Al}(\mathrm{OH})_{3}\right)$ and OVA-sensitized offspring (rats that received s.c. $200 \mu \mathrm{g}$ $\left.\mathrm{OVA}+8 \mathrm{mg} \mathrm{Al}(\mathrm{OH})_{3}\right)$. On day 14 , all the offspring were intratracheally injected with OVA $(0.4 \mathrm{ml}$ of $0.25 \%$ solution). Bronchoalveolar lavage was then performed at $48 \mathrm{~h}$ after OVA instillation. Another two groups of offspring were intratracheally injected with sterile saline $(0.4 \mathrm{ml})$ and their airways were washed with PBS buffer (as stated above) at $48 \mathrm{~h}$ post-saline injection. Leucocyte counts of the bronchoalveolar lavage fluid were then done.

\section{Statistics}

All data are expressed as mean and S.E.M. Data were analysed with the use of analysis of variance (ANOVA) followed by Duncan's test for multiple comparisons, and $t$-test for two independent samples. Significance was defined at the 0.05 level.

\section{Results}

\section{Bronchoalveolar lavage (BAL) fluid analysis in the naive offspring}

Bronchoalveolar lavage fluid from naive offspring revealed the presence of $0.7 \pm 0.1 \times 10^{6}$ and $0.6 \pm 0.1 \times 10^{6}$ leukocytes/BAL for non-stressed and prenatal-stressed, respectively, virtually all mononuclear cells $(n=6)$.

\section{Bronchoalveolar lavage fluid analysis both in non-sensitized and ovalbumin (OVA)-sensitized offspring}

In the non-sensitized offspring groups, no significant difference in the total leukocyte influx was observed between non-stressed and prenatal-stressed offspring (Table 1). In addition, the counts in neutrophils and mononuclear cells in the non-stressed offspring group did not differ significantly from those of prenatal-stressed offspring group (Table 1). Eosinophils were virtually absent in the non-sensitized animals, either in non-stressed or prenatal-stressed offspring.

In the OVA-sensitized offspring groups, a significant increase $(P<0.05)$ in total leukocyte infiltration was observed in non-stressed offspring (Fig. 1) compared to the non-sensitized, non-stressed group (Table 1). This was associated with a marked recruitment of eosinophils $(P<0.05)$ without a significant effect on 
Table 1. Total and differential leukocyte counts in the bronchoalveolar lavage (BAL) fluid from non-sensitized offspring rats, either non-stressed or prenatally stressed ones

\begin{tabular}{lcc}
\hline Cell type & \multicolumn{2}{c}{ Leukocytes $\left(\times 10^{6} / \mathrm{BAL}\right)$} \\
\cline { 2 - 3 } & Non-stressed & Prenatal-stressed \\
\hline $\mathrm{TL}$ & $1.45 \pm 0.2$ & $1.39 \pm 0.3$ \\
$\mathrm{NE}$ & $0.01 \pm 0.0$ & $0.12 \pm 0.1$ \\
$\mathrm{MN}$ & $1.44 \pm 0.2$ & $1.27 \pm 0.2$ \\
\hline
\end{tabular}

$\mathrm{TL}$, total leukocytes; NE, neutrophils; $\mathrm{MN}$, mononuclear cells. The results represent the mean \pm S.E.M. of nine to 18 rats.

the influx of neutrophils and mononuclear cells (Fig. 1). However, the total leukocyte influx was increased by $50 \%(P<0.05)$ in the prenatal-stressed offspring group as compared to the non-stressed one (Fig. 1). This was associated with a marked increase $(P<0.05)$ in the counts of both eosinophils and mononuclear cells without a significant effect on the neutrophils counts (Fig. 1).

In a separate group of both non-sensitized and OVAsensitized female offspring, either non-stressed or prenatal-stressed ones, $0.4 \mathrm{ml}$ of sterile saline (instead of OVA) was intratracheally injected into the animals and BAL evaluated $48 \mathrm{~h}$ post-saline injection. As expected, saline did not evoke a significant total leukocyte infiltration $(0.94 \pm 0.1,0.76 \pm 0.1,0.8 \pm 0.1$ and $0.92 \pm 0.1 \times 10^{6}$ total leukocytes/BAL in non-sensitized non-stressed, non-sensitized prenatal-stressed, OVA-sensitized non-stressed and OVA-sensitized prenatal-stressed offspring, respectively; $n=10$ ). The leukocyte infiltration was made up of $>99 \%$ mononuclear cells in all groups studied.

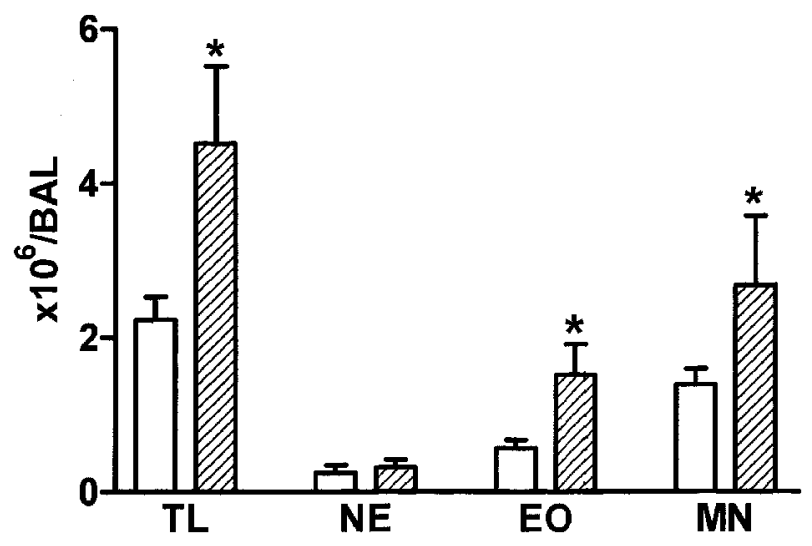

FIG. 1. Effects of mild chronic prenatal stress on total and differential (neutrophils, eosinophils and mononuclear cells) leukocyte counts in bronchoalveolar lavage (BAL) fluid from actively sensitized female offspring after $48 \mathrm{~h}$ following intratracheal injection of ovalbumin (OVA). Open columns represent non-stressed, whereas hatched columns represent prenatally stressed offspring. Each column represents the mean \pm S.E.M. $(n=11-15) .{ }^{*} P<0.05$ compared to their respective controls. $\mathrm{TL}$, total leukocytes; $\mathrm{NE}$, neutrophil; $\mathrm{MN}$, mononuclear cell; EO, eosinophil.

\section{Discussion}

The present study provides the first experimental evidence that chronic mild unpredictable prenatal stress produces a marked increase in the allergeninduced airway inflammation in the offspring, as evaluated by the large recruitment of eosinophils into their airways. Eosinophil accumulation in inflamed tissues plays a critical role in the allergic diseases, particularly bronchial asthma, since they synthesize and release a number of pro-inflammatory substances, including preformed granule proteins, oxygenderived toxic metabolites, arachidonic acid metabolites, platelet-activating factor and cytokines. ${ }^{2}$ Mononuclear cells, such as mast cells, macrophages and $\mathrm{T}$ lymphocytes, have also been implicated in the pathogenesis of asthma. A large infiltration of mononuclear cells into the airways of stressed (but not nonstressed) offspring was observed in our experimental model, indicating that antigen-induced airway inflammation in the stressed offspring is not specific for a particular leukocyte type but rather involves common mechanisms of cell recruitment.

The mechanisms by which chronic unpredictable mild prenatal stress exacerbate the allergen-induced airway inflammation are still unclear. However, it is well documented that circulating glucocorticoid hormones modulate allergic and non-allergic inflammation. Exacerbation of inflammatory responses as a consequence of endogenous corticosteroid suppression is usually observed in adrenalectomized animals. ${ }^{8}$ Maternal corticosterone crosses the placenta and inhibits hypothalamic-pituitary-adrenal (HPA) development in the fetuses. ${ }^{9,10}$ Furthermore, prenatal stress affects both development and activity of the HPA axis in the adult animals, ${ }^{11}$ and this may lead to an increase in the basal ACTH concentration, ${ }^{12}$ prolongation of stress-induced corticosterone secretion, ${ }^{13}$ and decrease in central corticosteroid receptors. ${ }^{14}$ This HPA hyperactivation in offspring from stressed mothers may cause its exhaustion and its inability to react properly to stress as a consequence of loss of neurofeedback control.

Corticosteroids and corticotrophin-releasing hormone $(\mathrm{CRH})$ are also important modulators of the immune system. ${ }^{15}$ Impairment of the normal HPA functioning increases both the susceptibility to inflammatory processes and immunological responses. ${ }^{16,20}$ Interestingly, this HPA dysfunction is also observed in endogenous depression, ${ }^{17}$ suggesting a correlation between inflammatory (and/or immunological) hyperactivity and depression. ${ }^{18}$ In this aspect, the stress schedule used in this study also aggravates the behavioral depression induced by the learned helplessness model. ${ }^{6}$ A recent study demonstrated that both cellular and specific humoral immune responses are enhanced in the offspring from prenatal stressed pregnant mothers. ${ }^{19}$ In conclusion, the 
clinical proposal that offspring from mothers exposed to stressful events during pregnancy are more susceptible to allergic diseases can be therefore clearly demonstrated in the rat using the experimental approach here reported. Our study provides a suitable experimental model to further investigate the mechanisms by which stress influences the triggering and development of allergic diseases.

ACKNOWLEDGEMENT. Paulo J. C. Nogueira was supported by CNPq.

\section{References}

1. Barnes PJ. Our changing understanding of asthma. Resp Med 1989: 83 S 17-23.

2. Kroegel C, Virchow J-C Jr, Luttmann W, Walker C, Warner JA. Pulmonary immune cells in health and disease: the eosinophil leucocyte Eur RespJ 1994: 7: 519-543.

3. Fischer S. Life stress, personal control, and the risk of disease. In: Coopes CL, eds Handbook of stress, Medicine and Health. Boca Raton, FL: CRC Press, 1995: 121-136.

4. Stott DN. Follow-up study from birth of the effects of prenatal stress. Dev Med Child Neurol 1973: 15: 770-787.

5. Willner P, Towell A, Sampson D, Sophokleas S, Muscat R. Reduction of sucrose preference by chronic unpredictable mild stress and its restoration by tricyclic antidepressants. Psychopharmacology 1987: 93: 358-364.

6. Secoli SR. Teixeira NA. Chronic prenatal stress affects development and behavioral depression in rats. Stress 1998: 2: 273-280.

7. Ferreira HHA, Bevilacqua E, Gagioti SM, Zanardo RCO, Teixeira CE, Sannomiya P, Antunes E, De Nucci G. Nitric oxide modulates eosinophil infiltration in antigen-induced airway inflammation in rats. Eur $J$ Pharmacol 1998: 358: 253-259.

8. Garcia-Leme, J. Hormones and Inflammation. Boca Raton, FL: CRC Press, 1989.
9. Zarrow MX, Philpott JE, Denenberg VH. Passage of ${ }^{14} \mathrm{C}$-4-corticosterone from the rat mother to the foetus and neonate. Nature 1970: 226: 1058-1059.

10. Ward IL, Weiz J. Differential effects of maternal stress on circulating levels of corticosterone, progestone and testosterone in male and female rat fetuses and their mothers. Endocrinology 1984: 114: 1635-1644.

11. Fameli M, Kitraki E. Stylianopoulou F. Effects of hyperactivity of the maternal hypothalamic-pituitary-adrenal (HPA) axis during pregnancy on the development of the HPA axis and brain monoamines of the offspring. Int J Dev Neurosci 1994: 12: 651-659.

12. MacCornick CM, Smythe JW, Sharma S, Meaney MJ. Sex-specific effects of prenatal stress on hypothalamic-pituitary-adrenal responses to stress and brain glucocorticoid receptor density in adult rats. Dev Brain Res 1995: 84: 55-61.

13. Weinstock M, Matlina E, Maos GI, Rosen H, McEwen B. Prenatal stress selectively alters the reactivity of the hypothalamic-pituitary-adrenal system in the female rats. Brain Res 1992: 595: 195-198.

14. Maccari S, Piazza PV, Kabbay M, Barbazangs A, Simon H, Le Moal M. Adoption reverses the long-term impairment in glucocorticoid feedback induced by prenatal stress. $J$ Neurosci 1995: 15: 110-116.

15. McEwen B, Biron C, Brunson K, Builoch K, Chambers W, Dhabhar F Goldfarbs R, Kitson R, Miller A, Spencer R, Weiss J. The role of adrenocorticoids as modulators of immune function in health and disease: neural, endocrine and immune interaction. Brain Res Rev 1997 23: 79-99.

16. Steinberg EM, Licino J. Overview of neuroimmune stress interactions implications for susceptibility to inflammatory disease. In: Stress basic mechanisms and clinical implications. Ann NY Acad Sci 1995: 771: $364-371$.

17. Gold PW, Goodwin FK, Chrousos GP. Clinical and biochemical manifestation of depression: relation to the neurobiology of stress (part 1 and 2). New Engl J Med 1988: 319: 348-420.

18. ConnorTJ, Leonard BE. Depression, stress and immunological activation the role of cytokines in depressive disorders. Life Sci 1998: 62 583-606.

19. Klein SL, Rager DR. Prenatal stress alters immune function in the offspring of rats. Dev Psychobiol 1995; 28: 321-336.

20. Harbuz MS, Jessop DS, Chowdrey HS, Blackwell JM, Larsen PJ, Lightman SL. Evidence for altered control of hypothalamic CRF in immunemediated disease. Ann NY Acad Sci 1995: 771: 449-458.

\section{Received 11 January 1999; accepted 11 February 1999}




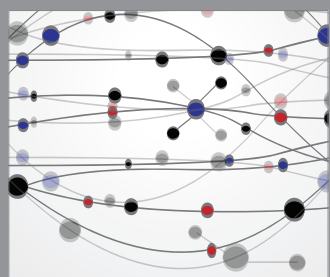

The Scientific World Journal
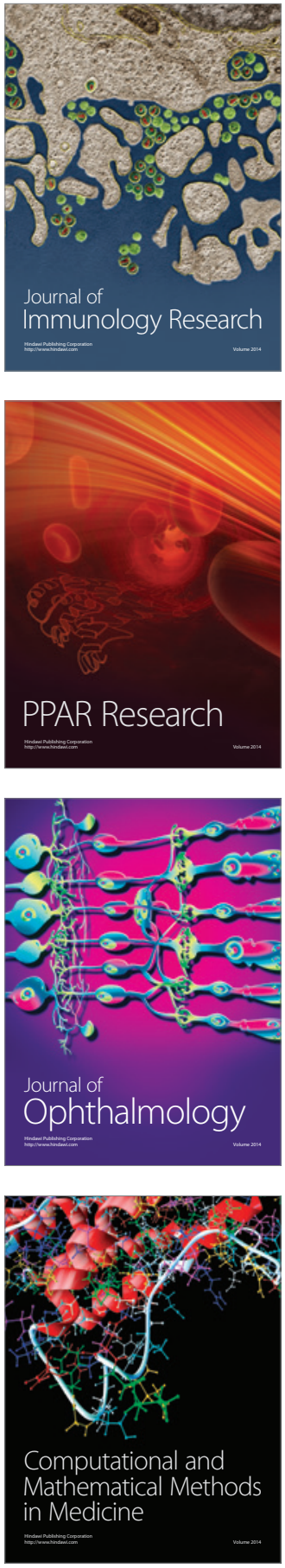

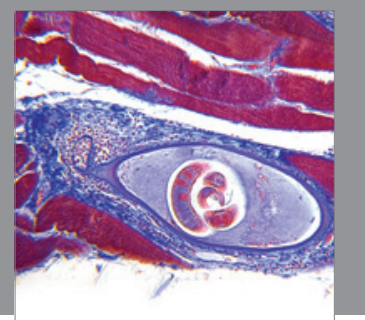

Gastroenterology

Research and Practice
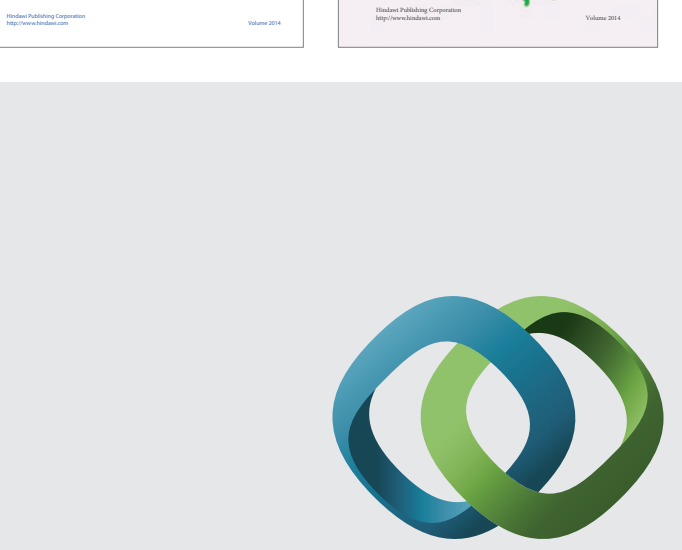

\section{Hindawi}

Submit your manuscripts at

http://www.hindawi.com
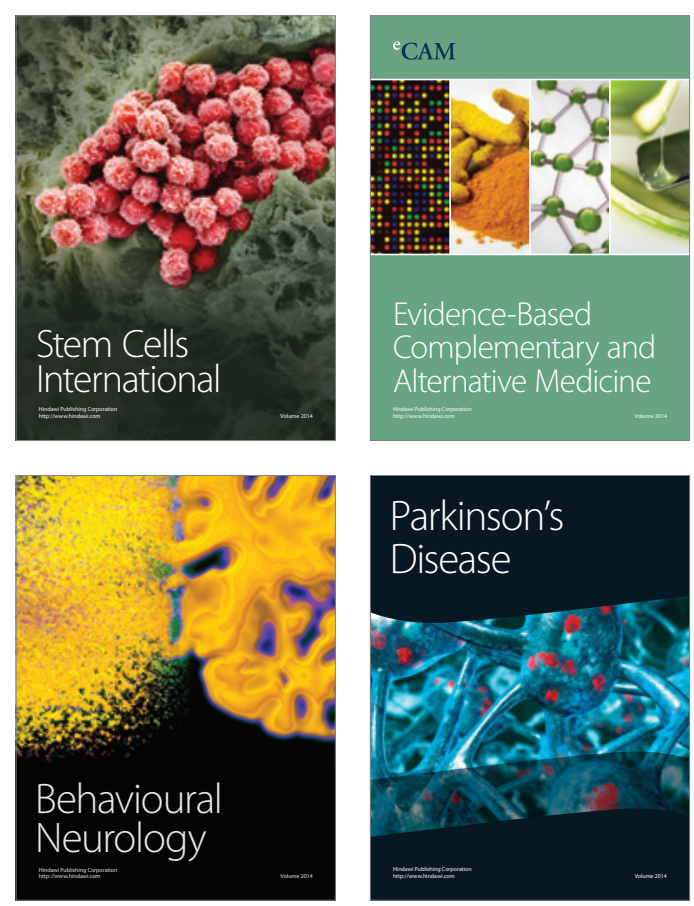

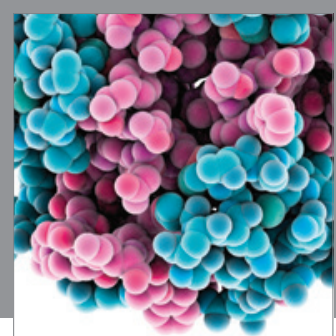

Journal of
Diabetes Research

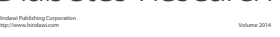

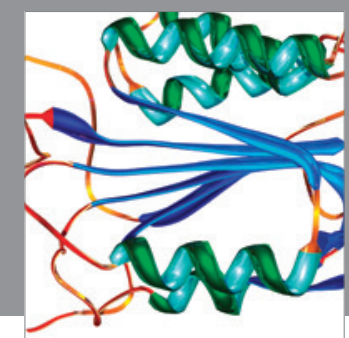

Disease Markers
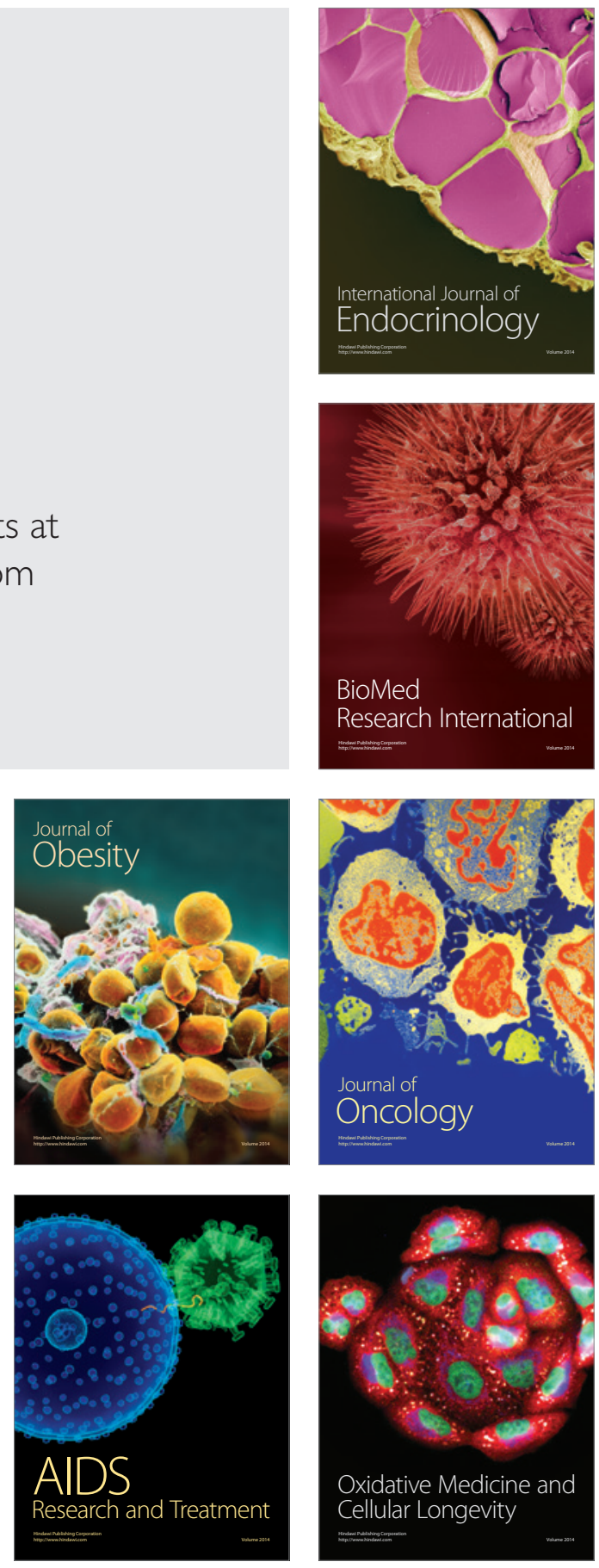